\title{
Anisotropic scattering and anomalous normal-state transport in a high-temperature superconductor
}

\author{
M. ABDEL-JAWAD ${ }^{1}$, M. P. KENNETT ${ }^{2}$, L. BALICAS ${ }^{3}$, A. CARRINGTON ${ }^{1}$, A. P. MACKENZIE ${ }^{4}$, \\ R. H. MCKENZIE ${ }^{5}$ AND N. E. HUSSEY'* \\ ${ }^{1}$ H. H. Wills Physics Laboratory, University of Bristol, Tyndall Avenue, Bristol BS8 1TL, UK \\ ${ }^{2}$ Physics Department, Simon Fraser University, 8888 University Drive, Burnaby, British Columbia V5A 1S6, Canada \\ ${ }^{3}$ National High Magnetic Field Laboratory, Florida State University, Tallahassee, Florida 32306, USA \\ ${ }^{4}$ School of Physics and Astronomy, University of St Andrews, St Andrews KY16 9SS, UK \\ ${ }^{5}$ Physics Department, University of Queensland, Brisbane 4072, Australia \\ *e-mail: n.e.hussey@bristol.ac.uk
}

Published online: 5 November 2006; doi:10.1038/nphys449

$\mathbf{T}$ he metallic state of high-temperature copper-oxide superconductors, characterized by unusual and distinct temperature dependences in the transport properties ${ }^{1-4}$, is markedly different from that of textbook metals. Despite intense theoretical efforts ${ }^{5-11}$, our limited understanding is impaired by our inability to determine experimentally the temperature and momentum dependence of the transport scattering rate. Here, we use a powerful magnetotransport probe to show that the resistivity and the Hall coefficient in highly doped $\mathrm{Tl}_{2} \mathrm{Ba}_{2} \mathrm{CuO}_{6+\delta}$ originate from two distinct inelastic scattering channels. One channel is due to conventional electronelectron scattering; the other is highly anisotropic, has the same symmetry as the superconducting gap and a magnitude that grows approximately linearly with temperature. The observed form and anisotropy place tight constraints on theories of the metallic state. Moreover, in heavily doped non-superconducting $\mathrm{La}_{2-x} \mathrm{Sr}_{x} \mathrm{CuO}_{4}$, this anisotropic scattering term is absent ${ }^{12}$, suggesting an intimate connection between the origin of this scattering and superconductivity itself.

The in-plane properties of layered metals can sometimes be obtained from measurements of out-of-plane quantities. For example, angular magnetoresistance oscillations (AMRO), which are angular variations in the interlayer resistivity $\rho_{\perp}$ induced by rotating a magnetic field $\mathbf{H}$ in a polar plane relative to the conducting layers, can provide detailed information on the shape of the in-plane Fermi surface (FS) in layered metals. Here we resolve for the first time, the momentum (k) and energy ( $\omega$ or $T$ ) dependence of the in-plane transport lifetime $\tau$ in an overdoped cuprate $\mathrm{Tl}_{2} \mathrm{Ba}_{2}\left(\mathrm{Ca}_{0}\right) \mathrm{Cu}_{1} \mathrm{O}_{6+\delta}$ (Tl2201) through advances, both experimental and theoretical, in the AMRO technique. Experimentally, we extend the temperature range of previous AMRO measurements on overdoped Tl2201 ${ }^{13}$ (with a superconducting transition temperature $T_{\mathrm{c}}=15 \mathrm{~K}$ ) by more than one order of magnitude. Theoretically, we derive a new general analytical expression for the interlayer conductivity $\sigma_{\perp}$ in a tilted $\mathbf{H}$ that incorporates basal-plane anisotropy. For $T>4 \mathrm{~K}$, the AMRO can only be explained by inclusion of an anisotropic scattering rate $1 / \tau$ whose anisotropy grows with $T$. Significantly, the anisotropy in $1 / \tau$ and its $T$ dependence up to $55 \mathrm{~K}$ can quantitatively account for both the robust linear-in- $T$ component to the in-plane resistivity $\rho_{\mathrm{ab}}$ and the $T$-dependent Hall coefficient $R_{\mathrm{H}}$ over the same temperature range ${ }^{14,15}$. These anomalous behaviours are not characteristic of a simple Fermi liquid, which is often the starting point for modelling overdoped cuprates. We discuss the consequences of these findings for our understanding of the normal-state transport in cuprates.

As described in the Supplementary Information, detailed azimuthal and polar-angle-dependent AMRO data were taken at $4.2 \mathrm{~K}$ and $45 \mathrm{~T}$ and fitted to the Shockley-Chambers tube integral form of the Boltzmann transport equation, modified for a quasitwo-dimensional (quasi-2D) metal ${ }^{16}$ (and assuming an isotropic mean-free-path $\ell$ ), to generate a full 3D parameterization of the FS wavevector $k_{\mathrm{F}}(\varphi, \theta)$ consistent with previous measurements ${ }^{13}$ (here $\varphi$ refers to in-plane angles and $\theta$ to polar angles out of the plane). Before studying the $T$ dependence of the scattering rate, a self-consistency check was carried out on the fitting procedure by varying $\mathbf{H}$ at a fixed temperature. The solid lines in Fig. 1a represent polar-angle-dependent changes in the interlayer resistivity $\Delta \rho_{\perp}(\theta) / \rho_{\perp}(H=0)$ at $4.2 \mathrm{~K}$ (normalized to the zerofield resistivity) for various fields $20 \mathrm{~T} \leq \mu_{0} H \leq 45 \mathrm{~T}$ at a fixed azimuthal orientation of the inclined sample $\phi=29^{\circ}$ (relative to the $\mathrm{Cu}-\mathrm{O}-\mathrm{Cu}$ bond direction) where all AMRO features are visible (here $\mu_{0}$ is the permittivity of free space). The magnetoresistance is determined by the magnitude of $\omega_{c} \tau$, the product of the 

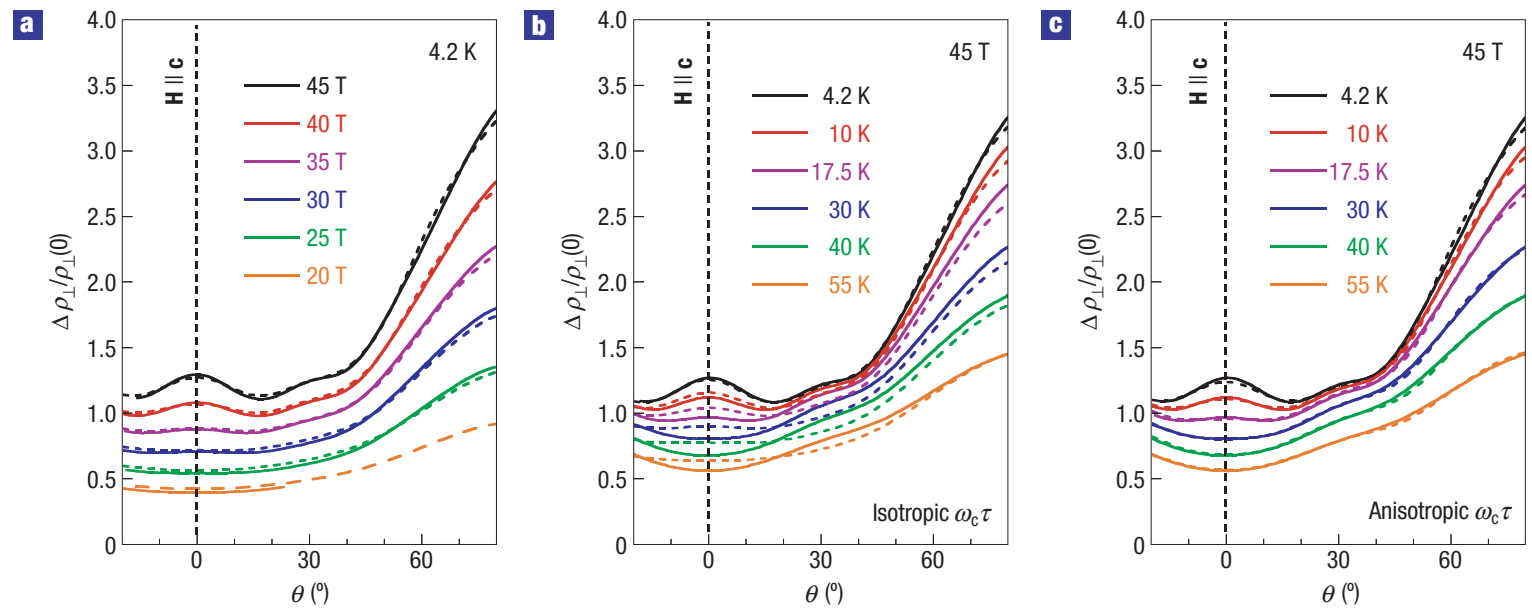

Figure 1 Field and temperature dependencies of the polar AMRO in overdoped TI2201 ( $\left.T_{\mathrm{c}}=15 \mathrm{~K}\right)$ at a fixed azimuthal direction $\phi=29^{\circ}$. a, Solid lines: normalized $\Delta \rho_{\perp}\left(\theta, \phi=29^{\circ}\right) / \rho_{\perp}(0)$ data for different field strengths. Dashed lines: simulated AMRO fits using the same $k_{\mathrm{mn}}$ coefficients as given in Supplementary Information, Fig. S1 and $\omega_{\mathrm{c}} \tau$ values scaled simply by the field scale (that is, $\left.\omega_{\mathrm{c}} \tau=0.4\left(\mu_{0} H / 45\right)\right)$. b. Solid lines: normalized $\Delta \rho_{\perp}\left(\theta, \phi=29^{\circ}\right) / \rho_{\perp}(0)$ data at $45 \mathrm{~T}$ for different temperatures between $4.2 \mathrm{~K}$ and $55 \mathrm{~K}$. Dashed lines: best least-squares fits using the same $k_{\mathrm{mn}}$ coefficients as given in Supplementary Information, Fig. $\mathrm{S} 1$ and assuming an isotropic $\omega_{\mathrm{c}} \tau$. $\mathbf{c}$, As b but with an anisotropic $\omega_{\mathrm{c}} \tau=\omega_{0} \tau_{0} /[1+\alpha \cos 4 \varphi]$.

cyclotron frequency and the transport lifetime. The dashed lines are simulated $\Delta \rho_{\perp}\left(\theta, \phi=29^{\circ}\right) / \rho_{\perp}(0)$ curves produced simply by scaling $\omega_{\mathrm{c}} \tau\left(=0.4\left(\mu_{0} H / 45\right)\right)$ whilst keeping all other parameters fixed at their $45 \mathrm{~T}$ values. The data scale very well, implying that the isotropic formalism ${ }^{16}$ remains valid with decreasing $H$ and that no additional angular dependence appears owing to the presence of inhomogeneous superconducting regions (with different $T_{\mathrm{c}}$ values) or anomalous vortex-liquid phases ${ }^{17}$.

Figure $1 \mathrm{~b}$ shows the temperature dependence of $\Delta \rho_{\perp}(\theta, \phi=$ $\left.29^{\circ}\right) / \rho_{\perp}$ up to $55 \mathrm{~K}\left(\mu_{0} H=45 \mathrm{~T}\right)$. Remarkably, AMRO features remain discernible at all temperatures, in particular the kink around $\theta=30^{\circ}$. Comparison of the data in Fig. 1a and $\mathrm{b}$ reveals that the AMRO evolve differently depending on whether $\omega_{c} \tau$ is reduced by decreasing $H$ or by increasing $T$. In the former case, both the peak at $\mathbf{H} \| \mathbf{c}$ and the peak at intermediate angles diminish at approximately the same rate, whereas in the latter, the intermediate peak is found to survive up to much higher temperatures. The dashed lines in Fig. $1 \mathrm{~b}$ show the best least-squares fits to the data assuming all parameters except the product $\omega_{\mathrm{c}} \tau$ remain constant up to $55 \mathrm{~K}$. These fits are clearly inferior to those in Fig. 1a.

To proceed, we relax the constraint that $\omega_{\mathrm{c}} \tau$ remains isotropic at all temperatures and generalize the expression ${ }^{16}$ for $\sigma_{\perp}$ to incorporate basal-plane anisotropy in the relevant parameters. We first parameterize the Fermi velocity as $\mathbf{v}_{\mathrm{F}}(\varphi)=v_{\mathrm{F}}^{0}(1+\beta \cos 4 \varphi)$, where $\beta$ is the anisotropy in $v_{\mathrm{F}}$, and the variation of $\omega_{\mathrm{c}}$ around the FS as

$$
\omega_{\mathrm{c}}(\varphi, \theta)=e \mu_{0} H \cos \theta \frac{\mathbf{k}_{\mathrm{F}}(\varphi) \cdot \mathbf{v}_{\mathrm{F}}(\varphi)}{\hbar k_{\mathrm{F}}(\varphi)^{2}} .
$$

The generalized expression for $\sigma_{\perp}$ then becomes

$$
\begin{aligned}
\sigma_{\perp}= & \frac{e^{2}}{4 \pi^{3} \hbar^{2}} \frac{1}{1-P} \int_{-\pi / d}^{\pi / d} \mathrm{~d} k_{\perp} \int_{0}^{2 \pi} \mathrm{d} \varphi_{2} \frac{e \mu_{0} H \cos \theta}{\omega_{\mathrm{c}}\left(\varphi_{2}\right)} \\
& \times \int_{\varphi_{2}-2 \pi}^{\varphi_{2}} \mathrm{~d} \varphi_{1} v_{\perp}\left(\varphi_{2}, k_{\perp}\right) v_{\perp}\left(\varphi_{1}, k_{\perp}\right) \frac{G\left(\varphi_{2}, \varphi_{1}\right)}{\omega_{\mathrm{c}}\left(\varphi_{1}\right)}
\end{aligned}
$$

where $k_{\perp}$ is the $c$-axis reciprocal lattice vector, $v_{\perp}$ is the interlayer velocity, $\hbar$ is the reduced Planck's constant, $d$ is the interlayer spacing $(=1.16 \mathrm{~nm}$ for Tl2201), $P=G(2 \pi, 0)$ is the probability that an electron makes a complete orbit of the FS without being scattered and

$$
G\left(\varphi_{2}, \varphi_{1}\right)=\exp \left(-\int_{\varphi_{1}}^{\varphi_{2}} \frac{\mathrm{d} \varphi}{\omega_{c}(\varphi) \tau(\varphi)}\right)
$$

This formalism holds irrespective of whether hopping is coherent or weakly incoherent (that is, when $\hbar / \tau>2 t_{\perp}$, the interlayer hopping energy, and $v_{\perp}$ is ill-defined $)^{18}$. In the latter case, AMRO arise from differences in Aharonov-Bohm phases acquired in hopping between layers for positions $\varphi_{1}$ and $\varphi_{2}$ on the $\mathrm{FS}^{19}$.

Consistent with the tetragonal symmetry of Tl2201, we write $1 / \tau(\varphi)=(1+\alpha \cos 4 \varphi) / \tau_{0}$. Although no unique and independent determination of the various anisotropy parameters can be made from fits of theoretical curves to AMRO data alone, certain features of the data tightly constrain the parametrization, in particular the FS parameters defining $k_{\mathrm{F}}(\varphi, \theta)$ (ref. 13). Furthermore, as there is no experimental evidence to suggest changes in the FS topography with temperature, we fix these parameters to their values at $4.2 \mathrm{~K}$. Similarly, $\beta$, the anisotropy in $v_{\mathrm{F}}$, is assumed to be constant. Finally, to minimize the number of fitting parameters, we assume that $\omega_{\mathrm{c}}$ is isotropic $\left(=\omega_{0}\right)$ within the basal plane. Thus, we can provisionally ascribe the evolution of the AMRO uniquely to changes in $1 / \omega_{0} \tau(\varphi)$ and extract $1 / \omega_{0} \tau_{0}(T)$ and $\alpha(T)$ from fits to the data at different temperatures. The best least-square fits are shown in Fig. 1c. The quality of the fits at all $T$ is clearly much improved with just the inclusion of $\alpha(T)$, the anisotropy in the scattering rate. Moreover, the subsequent fitting to the in-plane transport data is sufficiently good (see below) that the introduction of additional parameter(s), for example, to account for any possible $T$ dependence in $\beta$, seems to be unnecessary (for more details see the Supplementary Information).

The consequences of the above analysis are examined in Fig. 2. To aid our discussion, we show schematically in Fig. 2a the in-plane geometry of various relevant entities with respect to the $2 \mathrm{D}$ projection of the FS of overdoped Tl2201 (red curve in Fig. 2a). The purple line represents the $d$-wave superconducting gap, whereas 

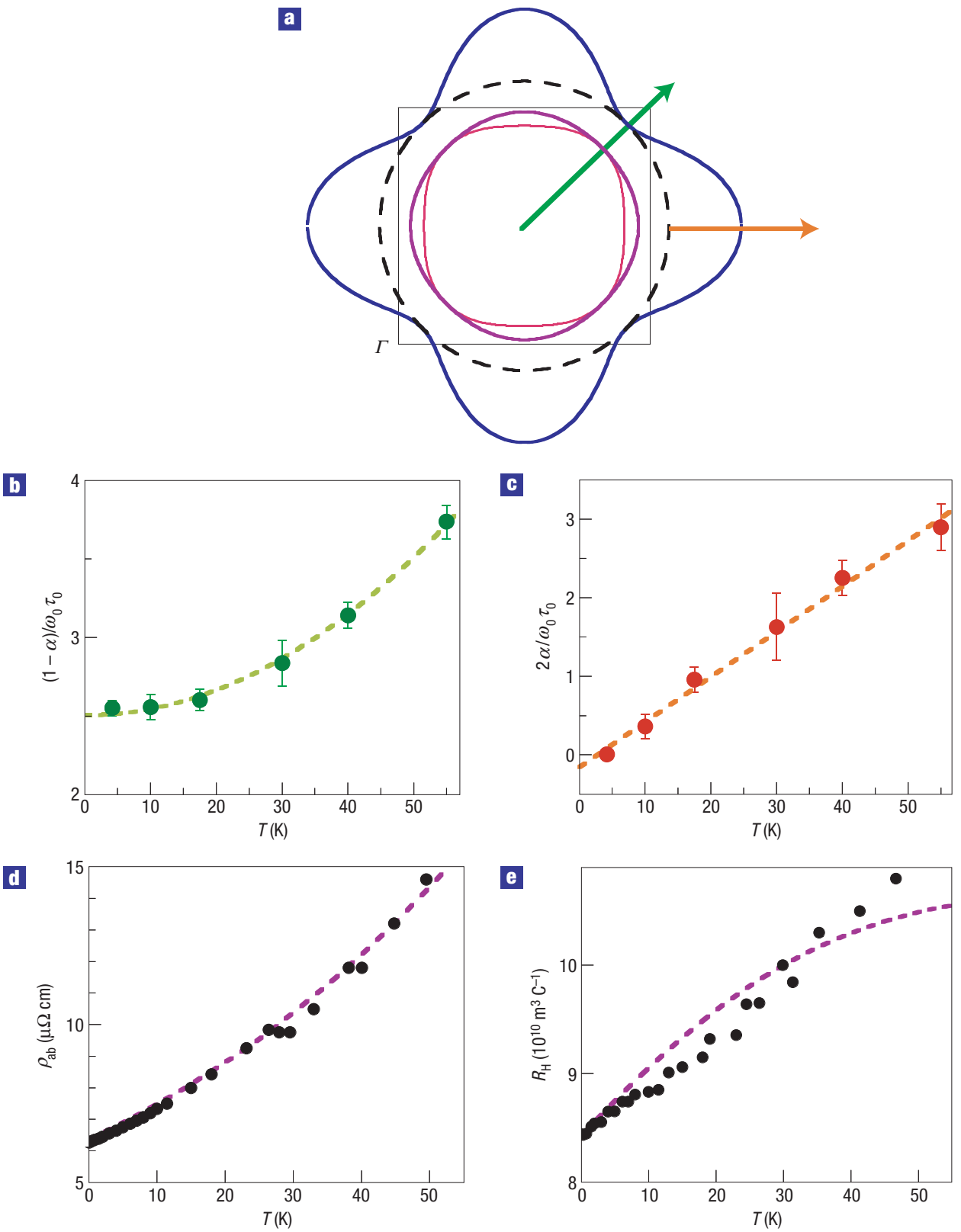

Figure 2 Determination of the in-plane transport coefficients from 45 T polar AMRO. a, Red curve: schematic 2D projection of the FS of overdoped TI2201. Purple curve: schematic representation of the $d$-wave superconducting gap. Blue curve: geometry of $\left(\omega_{\mathrm{c}} \tau\right)^{-1}(\varphi)$. Black dashed line: isotropic part of $\left(\omega_{\mathrm{c}} \tau\right)^{-1}(\varphi)$. $\mathbf{b}, T$ dependence of $(1-\alpha) / \omega_{0} \tau_{0}$, that is, the isotropic component of $\left(\omega_{\mathrm{c}} \tau\right)^{-1}(\varphi)$ and sole contribution along the 'nodal' region indicated by the green arrow in $\mathbf{a}$. The green dashed curve is a fit to $A+B T^{2}$. c, $T$ dependence of $2 \alpha / \omega_{0} \tau_{0}$, that is, the anisotropic component of $\omega_{\mathrm{c}} \tau^{-1}(\varphi)$ and the additional contribution that is maximal along the 'anti-nodal' direction indicated by the orange arrow in a. The orange dashed curve is a fit to $C+D T$. d, Black circles: $\rho_{\mathrm{ab}}(T)$ data for overdoped TI2201 $\left(T_{\mathrm{c}}=15 \mathrm{~K}\right)$ extracted from ref. 14 . Purple dashed curve: simulation of $\rho_{\mathrm{ab}}(T)$ from parameters extracted from our AMRO analysis. To aid comparison, $1.9 \mu \Omega \mathrm{cm}$ have been subtracted from the simulated data. (It is not unreasonable to expect different crystals to have different residual resistivities.) e, Black circles: $R_{H}(T)$ data for the same crystal ${ }^{14}$. Purple dashed curve: simulation of $R_{\mathrm{H}}(T)$ from parameters extracted from our AMRO analysis. In this case, no adjustments have been made. $\rho_{\mathrm{ab}}(T)$ and $R_{\mathrm{H}}(T)$ were calculated using the Jones-Zener expansion of the linearized Boltzmann transport equation for a quasi-2D FS (ref. 7). Note that using (1), we can re-express the expressions in ref. 7 solely in terms of parameters extracted from our analysis. The error bars in $\mathbf{b}, \mathbf{c}$ are the covariance matrix of the multidimensional least-squares fitting routine.

the blue solid line shows our deduced geometry of $1 / \omega_{\mathrm{c}} \tau(\varphi)$ (as governed by the sign of $\alpha$ ), its maximum being at $\varphi=0^{\circ}$. Note that the scattering anisotropy and the superconducting order parameter have the same symmetry. This is consistent with earlier azimuthal AMRO data ${ }^{20}$ but contrasts with recent angle-resolved photoemission spectroscopy (ARPES) measurements ${ }^{21}$. However, we note that the ARPES-derived scattering rate is one order of magnitude larger, suggesting that the two probes are not measuring the same quantity.

To give our anisotropic function for $\omega_{\mathrm{c}} \tau$ more physical meaning, we re-express $(1+\alpha \cos 4 \varphi) / \omega_{0} \tau_{0}$ as $(1-\alpha) / \omega_{0} \tau_{0}+$ $\left(2 \alpha / \omega_{0} \tau_{0}\right) \cos ^{2} 2 \varphi$. The isotropic part $(1-\alpha) / \omega_{0} \tau_{0}$ (black dashed 
line in Fig. 2a) is the sole contribution along the diagonal 'nodal' direction (indicated by the green arrow) where the pairing gap vanishes. The $T$ dependence of $(1-\alpha) / \omega_{0} \tau_{0}$ is plotted in Fig. $2 b$ and as shown by the dashed line, follows a simple quadratic law $\left(A+B T^{2}\right)$. In contrast, the anisotropic component $2 \alpha / \omega_{0} \tau_{0}$, maximal in the direction given by the orange arrow in Fig. $2 \mathrm{a}$ and plotted in Fig. 2c, is seen to grow approximately linearly with temperature, this linearity extending at least down to $4.2 \mathrm{~K}$.

To our knowledge, this is the first quantitative determination of the momentum and temperature dependence of the in-plane mean-free-path in cuprates. Together with the complete FS topology, this is all we need in principle to calculate the various coefficients of the in-plane conductivity tensor. Figure $2 \mathrm{~d}$ shows $\rho_{\mathrm{ab}}(T)$ as determined from our analysis, superimposed on published data for overdoped T12201 at the same doping level (with the superconductivity suppressed by a large magnetic field $)^{14}$. The form of $\rho_{\mathrm{ab}}(T)$, in particular the strong $T$-linear component below $10 \mathrm{~K}$ and the development of supra-linear behaviour above this temperature, is extremely well reproduced by the model. The corresponding $R_{\mathrm{H}}(T)$ is shown in Fig. 2e. Significantly, the absolute change in anisotropy in $\left(\omega_{0} \tau\right)^{-1}(\varphi, T)$ can account fully for the rise in $R_{\mathrm{H}}(T)$, at least up to $40 \mathrm{~K}$. Above $40 \mathrm{~K}$, the simulation has a slightly weaker $T$ dependence, possibly due to the increased disorder in the AMRO sample, known to weaken the overall $T$ dependence of $R_{\mathrm{H}}(T)$ in cuprates ${ }^{2}$, and/or the emergence of vertex corrections that manifest themselves only in the in-plane transport ${ }^{22}$. Overall however, the same parametrization of $1 / \omega_{0} \tau(\varphi, T)$ described in Fig. $2 \mathrm{~b}, \mathrm{c}$ gives an excellent account, not only of the evolution of the AMRO signal (Fig. 1c), but also of the 'anomalous' transport behaviour. Given the gradual evolution of the transport properties in T12201 with doping $^{23}$, we believe these findings will be relevant to crystals with higher $T_{\mathrm{c}}$ values.

We now discuss the implications of our results for existing theories of transport in high- $T_{c}$ cuprates. Several contrasting approaches dominate much current thinking: Anderson's resonantvalence-bond picture ${ }^{24}$, marginal Fermi-liquid phenomenology ${ }^{6}$ and models based on fermionic quasiparticles that invoke specific (anisotropic) scattering mechanisms within the basal plane owing either to anisotropic electron-electron (possibly Umklapp) scattering $^{7}$ or coupling to a singular bosonic mode, be that of $\operatorname{spin}^{8,9}$, charge $e^{10}$ or superconducting fluctuations ${ }^{11}$. Our analysis clearly supports those models in which anisotropy in the inelastic part of $\ell(\mathbf{k})$ is responsible for the anomalous $R_{\mathrm{H}}(T)$. Empirically, both $R_{\mathrm{H}}(T)$ and the $T$-linear component of $\rho_{\mathrm{ab}}(T)$ are derived from a $T$-linear anisotropic scattering term that is maximal along the $\mathrm{Cu}-\mathrm{O}-\mathrm{Cu}$ bond direction. The magnitude of the anisotropy is large, even at such an elevated doping level. At $T=55 \mathrm{~K}$, for example, $\ell(\mathbf{k})$ varies by a factor of two around the in-plane FS. Significantly, in non-superconducting cuprates, $\rho_{\mathrm{ab}}(T) \propto T^{2}$ at low temperatures with no evidence of a $T$-linear term ${ }^{12,23}$. This implies that the development of superconductivity (from the overdoped side) is closely correlated with the appearance of the $T$-linear resistivity and anisotropic inelastic scattering, a correlation that will be explored further in future studies. (Recall that $1 / \tau(\varphi)$ also has the same angular dependence as the superconducting gap.)

Our analysis implies the presence of (at least) two inelastic scattering channels in the current response of superconducting cuprates. Recent ARPES measurements ${ }^{25}$ on $\mathrm{Bi}_{2} \mathrm{Sr}_{2} \mathrm{CaCu}_{2} \mathrm{O}_{8-\delta}$ also found evidence for two contributions to the quasiparticle (singleparticle) scattering rate; one quadratic in $\omega$ and one linear in $\omega$ that develops a kink below $T_{c}$. A scattering process that is quadratic in both temperature and frequency is characteristic of electron-electron scattering. Given that the Hall conductivity is dominated by those regions (in this case, the nodal regions) where scattering is weakest, we thereby ascribe the $T^{2}$ dependence of the inverse Hall angle $\cot \theta_{\mathrm{H}}$ in cuprates to such scattering. Recall that $\cot \theta_{\mathrm{H}}$ does not vary markedly across the cuprate phase diagram ${ }^{8,23}$ and so the strength of electron-electron scattering seems largely doping independent.

The second term (seen by ARPES) has been attributed to scattering off a bosonic mode, though its origin and its relevance to high- $T_{c}$ superconductivity remain subjects of intense debate ${ }^{26}$. Possible candidates include phonons, $d$-wave pairing fluctuations, spin and charge fluctuations but as all, bar phonons, seem to vanish in heavily overdoped non-superconducting cuprates ${ }^{11,27,28}$, it is difficult to single one out at this stage. For a bosonic mode to be the source of anisotropic scattering revealed by AMRO however, the continuation of its linear $T$-dependence to very low temperatures is highly constraining, requiring as it does the presence of an extremely low energy scale. An alternative origin for this k-space anisotropic scattering is real-space (correlated) inhomogeneity. Indeed in underdoped cuprates, intense anisotropic scattering exists alongside gross inhomogeneity and checkerboard charge order $^{29}$. As the Mott insulator is approached, the degree of inhomogeneity grows and the simple anisotropic metal evolves into a more exotic 'nodal' metallic state in which the FS is reduced to a series of Fermi arcs in those (nodal) regions where scattering is weakest ${ }^{30}$. We stress again though that both the anisotropy and the $T$ dependence of the scattering need to be accounted for. Nevertheless, the stronger $T$-linear behaviour seen in $\rho_{\mathrm{ab}}$ as maximum $T_{\mathrm{c}}$ is approached points to an increase in the anomalous term with lower doping, and the connection between the anisotropy in the under- and overdoped regimes is clearly an important avenue for future research.

Finally, this work demonstrates that AMRO can be an extremely powerful probe of intralayer anisotropies in layered metals, beyond mere determination of the FS. The formalism and procedure we have used here could be applied to a host of other layered correlated metals, for example, molecular superconductors and ruthenates, to establish whether anisotropic scattering also plays an important role in the unconventional behaviour observed in these systems.

Received 24 July 2006; accepted 4 October 2006; published 5 November 2006.

\section{References}

1. Gurvitch, M. \& Fiory, A. T. Resistivity of $\mathrm{La}_{1.825} \mathrm{Sr}_{0.175} \mathrm{CuO}_{4}$ and $\mathrm{YBa}_{2} \mathrm{Cu}_{3} \mathrm{O}_{7}$ to $1100 \mathrm{~K}$ : Absence of saturation and its implications. Phys. Rev. Lett. 59, 1337-1340 (1987).

2. Chien, T. R., Wang, Z. Z. \& Ong, N. P. Effect of $Z n$ impurities on the normal state Hall angle in single crystal $\mathrm{YBa}_{2} \mathrm{Cu}_{3-x} \mathrm{Zn}_{x} \mathrm{O}_{7-\delta}$. Phys. Rev. Lett. 67, 2088-2091 (1991).

3. Hwang, H. Y. et al. Scaling of the temperature dependent Hall effect in $\mathrm{La}_{2-x} \mathrm{Sr}_{x} \mathrm{CuO}_{4}$. Phys. Rev. Lett. 72, 2636-2639 (1994)

4. Harris, J. M. et al. Violation of Kohler's rule in the normal-state magnetoresistance of $\mathrm{YBa}_{2} \mathrm{Cu}_{3} \mathrm{O}_{7-\delta}$ and $\mathrm{La}_{2-x} \mathrm{Sr}_{x} \mathrm{CuO}_{4}$. Phys. Rev. Lett. 75, 1391-1394 (1995).

5. Lee, P. A., Nagaosa, N. \& Wen, X.-G. Doping a Mott insulator: Physics of high-temperature superconductivity. Rev. Mod. Phys. 78, 17-85 (2006)

6. Varma, C. M. et al. Phenomenology of the normal state of $\mathrm{Cu}-\mathrm{O}$ high-temperature superconductors. Phys. Rev. Lett. 63, 1996-1999 (1989).

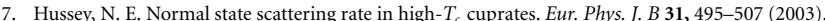

8. Carrington, A., Mackenzie, A. P., Lin, C. T. \& Cooper, J. R. Temperature dependence of the Hall angle in single-crystal $\mathrm{YBa}_{2}\left(\mathrm{Cu}_{1-x} \mathrm{Co}_{x}\right)_{3} \mathrm{O}_{7-\delta}$. Phys. Rev. Lett. 69, 2855-2878 (1992).

9. Monthoux, P. \& Pines, D. Spin-fluctuation-induced superconductivity and normal-state properties of $\mathrm{YBa}_{2} \mathrm{Cu}_{3} \mathrm{O}_{7}$. Phys. Rev. B 49, 4261-4278 (1992).

10. Castellani, C., di Castro, C. \& Grilli, M. Singular quasiparticle scattering in the proximity of charge instabilities. Phys. Rev. Lett. 75, 4650-4653 (1995).

11. Ioffe, L. B. \& Millis, A. J. Zone-diagonal-dominated transport in high- $T_{\mathrm{c}}$ cuprates. Phys. Rev. B 58, 11631-11638 (1998).

12. Nakamae, $\mathrm{S}$. et al. Electronic ground state in heavily overdoped non-superconducting $\mathrm{La}_{2-x} \mathrm{Sr}_{x} \mathrm{CuO}_{4}$. Phys. Rev. B 68, 100502 (2003).

13. Hussey, N. E. et al. A coherent three-dimensional Fermi surface in a high transition temperature superconductor. Nature 425, 814-817 (2003)

14. Mackenzie, A. P., Julian, S. R., Sinclair, D. C. \& Lin, C. T. Normal state magnetotransport in superconducting $\mathrm{Tl}_{2} \mathrm{Ba}_{2} \mathrm{CuO}_{6+\delta}$ to millikelvin temperatures. Phys. Rev. B 53, 5848-5855 (1996).

15. Proust, C. et al. Heat transport in a strongly overdoped cuprate: Fermi liquid and a pure $d$-wave BCS superconductor. Phys. Rev. Lett. 89, 147003 (2002).

16. Yagi, T., Iye, Y., Osada, T. \& Kagoshima, S. Semiclassical interpretation of the angular-dependent oscillatory magnetoresistance in quasi-2D systems. J. Phys. Soc. Japan 59, 3069-2072 (1990).

17. Geshkenbein, V. B., Ioffe, L. B. \& Millis, A. J. Theory of the resistive transition in overdoped $\mathrm{Tl}_{2} \mathrm{Ba}_{2} \mathrm{CuO}_{6+8}$ : implications for the vortex viscosity and the quasiparticle scattering rate in high- $T_{\mathrm{c}}$ superconductors. Phys. Rev. Lett. 80, 5778-5791 (1998). 
18. Moses, P. \& McKenzie, R. H. Incoherent interlayer transport and angular-dependent magneto-resistance oscillations in layered metals. Phys. Rev. Lett. 81, 4492-4495 (1998).

19. Cooper, B. K. \& Yakovenko, V. M. Interlayer Aharonov-Bohm interference in tilted magnetic fields in quasi-one-dimensional organic conductors. Phys. Rev. Lett. 96, 037001 (2006).

20. Hussey, N. E. et al. Angular dependence of the $c$-axis normal state magnetoresistance in single crystal $\mathrm{Tl}_{2} \mathrm{Ba}_{2} \mathrm{CuO}_{6+\delta}$. Phys. Rev. Lett. 76, 122-125 (1996).

21. Platé, M. et al. Fermi surface and quasiparticle excitations of overdoped $\mathrm{Tl}_{2} \mathrm{Ba}_{2} \mathrm{CuO}_{6+\delta}$. Phys. Rev. Lett. 95, 077001 (2005)

22. Sandeman, K. \& Schofield, A. J. Model of anisotropic scattering in a quasi-two-dimensional metal. Phys. Rev. B 63, 094510 (2001).

23. Manako, T., Kubo, Y. \& Shimakawa, T. Transport and structural study of $\mathrm{Tl}_{2} \mathrm{Ba}_{2} \mathrm{CuO}_{6+\delta}$ single crystals prepared by the KCl flux method. Phys. Rev. B 46, 11019-11024 (1992).

24. Anderson, P. W. The resonating valence bond state in $\mathrm{La}_{2} \mathrm{CuO}_{4}$ and superconductivity. Science 235 $1196-1198$ (1987)

25. Kordyuk, A. A. et al. Manifestation of the magnetic resonance mode in the nodal quasiparticle lifetime of the superconducting cuprates. Phys. Rev. Lett. 92, 257006 (2004).

26. Wilson, J. A. Bosonic mode interpretation of novel scanning tunnelling microscopy and related experimental results, within boson-fermion modelling of cuprate high-temperature superconductivity. Phil. Mag. 84, 2183-2216 (2004).

27. Wakimoto, S. et al. Direct relation between the low-energy spin excitations and superconductivity of overdoped high- $T_{c}$ superconductors. Phys. Rev. Lett. 92, 217004 (2004).

28. Reznik, D. et al. Electron-phonon coupling reflecting dynamic charge inhomogeneity in copper-oxide superconductors. Nature 440, 1170-1173 (2006).
29. McElroy, K. M. et al. Coincidence of checkerboard charge order and antinodal state decoherence in strongly underdoped superconducting $\mathrm{Bi}_{2} \mathrm{Sr}_{2} \mathrm{CaCu}_{2} \mathrm{O}_{8+\delta}$. Phys. Rev. Lett. 94, 197005 (2005).

30. Civelli, M. et al. Dynamical break-up of the Fermi surface in a doped Mott insulator. Phys. Rev. Lett. 95, $106402(2005)$.

\section{Acknowledgements}

We thank J. C. Davis, L. P. Gor'kov, B. L. Gyorrfy, P. B. Littlewood, A. J. Schofield, N. Shannon and J. A. Wilson for helpful discussions. We also acknowledge technical support from V. Williams in the development of the two-axis rotator conceived by L.B. This work was supported by EPSRC and a co-operative agreement between the State of Florida and NSF.

Correspondence and requests for materials should be addressed to N.E.H.

Supplementary Information accompanies this paper on www.nature.com/naturephysics.

\section{Author contributions}

The crystals were supplied by A.C. and A.P.M., experiments were carried out by M.A.-J., L.B. and N.E.H. and the analysis was carried out by M.A.-J., M.P.K., R.H.M. and N.E.H.

Competing financial interests

The authors declare that they have no competing financial interests.

Reprints and permission information is available online at http://npg.nature.com/reprintsandpermissions/ 